\title{
Self-sustained current oscillations in superlattices and the van der Pol equation
}

\author{
Z. Z. Sun, ${ }^{1}$ J. P. Cao, ${ }^{1,2}$ Sun Yin,${ }^{1}$ Y. P. Wang, ${ }^{2}$ Y. Q. Wang, ${ }^{3}$ and X. R. Wang ${ }^{1, *}$ \\ ${ }^{1}$ Physics Department, The Hong Kong University of Science and Technology, Clear Water Bay, Hong Kong SAR, China \\ ${ }^{2}$ Institute of Physics \& Center for Condensed Matter Physics, \\ Chinese Academy of Sciences, Beijing 100080, P.R. China \\ ${ }^{3}$ Institute of Solid State Physics, Chinese Academy of Sciences, Hefei, P.R. China
}

(Dated: September 25, 2006)

\begin{abstract}
The connection between self-sustained current oscillations (SSCOs) in superlattices and the famous van der Pol (vdP) equation is established by mapping a widely used model of SSCOs to the $\mathrm{vdP}$ equation. Since this equation can describe an LCR circuit with a nonlinear resistor of negative differential resistance, we have obtained an equivalent electric circuit for superlattices in the SSCO regime. The origin of the equivalent inductance in superlattices is revealed.
\end{abstract}

PACS numbers: 73.50.Fq, 05.45.-a, 05.45.Xt

Following the original suggestions of Esaki and coworkers[1,2], there has been a great deal of experimental and theoretical work on the vertical transport of superlattices (SLs). One of the recent surprising discoveries is the self-sustained current oscillations (SSCOs) under dc bias[3, 4]. It has been shown[5,6] that SSCOs can be understood as the manifestations of limit cycle solutions around unstable fixed points. This picture provides also an explanation to the frequency locking observed in ac response[6].

A limit cycle is an isolated closed curve in a phase space. A closed curve is embedded in a two dimensional surface. Thus there are two and only two relevant variables for a limit cycle solution. The motion of the system on this surface can be described by a two-dimensional dynamic system. In another word, a periodic motion on a limit cycle can in principle be modelled by a second-order ordinary differential equation (ODE) if proper variables are used. A natural question is what is the corresponding second-order ODE for the SSCOs in SLs. It is well known that the so-called van der Pol (vdP) equation[7] can describe self-sustained oscillations in many natural dissipative systems such as chemical reactions and an LCR circuit with a nonlinear NDC under a dc bias. On the other hand, an SL has obviously both capacitor and resistor components. It has also a nonlinear resistor with NDC[1]. Furthermore, there are also proposals that SL structures may have inductor components[8]. Thus, it is legitimate to ask whether SSCOs in SLs are the same type of oscillations as that in a LCR circuit described by the vdP equation. This is the issue that we want to address. In this letter, we show that a widely used model for SSCOs in SLs can be indeed mapped into the vdP equation. Thus, in terms of oscillation, an SSCO in an $\mathrm{SL}$ is in the same class as that of the vdP equation.

We start from a discrete drift (DD) model widely used for SSCOs in SLs[9]. Consider an SL system of $N$ quan-

\footnotetext{
${ }^{*}$ To whom correspondence should be addressed. Electronic address: phxwan@ust.hk
}

tum wells. An external bias $U$ is applied between the two end wells. A current $I_{i-1}$ passes through the $i^{\text {th }}$ barrier under a given bias $V_{i}$. The system is governed by the discrete Poisson equations

$$
k\left(V_{i+1}-V_{i}\right)=n_{i}-N_{D}, \quad i=1,2, \ldots N,
$$

and the current continuity equations

$$
J=k \frac{\partial V_{i}}{\partial t}+I_{i-1}, \quad i=1,2, \ldots N+1
$$

where $n_{i}$ is the electric charge density in the $i^{t h}$ well, $N_{D}$ is doping concentration, and $k$ is a system parameter. A stead-state solution (SSS) may be unstable[9] if one chooses $I_{i}=n_{i} v_{i+1}\left(V_{i+1}\right)$, where $v_{i}$ is a phenomenological drift velocity through barrier $i$ which is, for simplicity, assumed to be a function of $V_{i}$ only. The constraint equation for $V_{i}$ is

$$
\sum_{i=1}^{N+1} V_{i}=U
$$

To close the equations, a suitable boundary condition is needed. It is reasonable $[5,6]$ to assume a constant $n_{0}$, $n_{0}=\delta N_{D}$ with $\delta$ as a model parameter. $k, U$, and $N_{D}$ can be set to 1 by choosing proper units for charge, length, and voltage. After eliminating $n_{i}$ through Eqs. (1), one can obtain a $N$-dimensional dynamic ODEs for $V_{i}$.

Although most experiments of SSCOs in SLs were done with about 40 wells, SSCOs were also observed in SLs with as few as 10 wells. Obviously, larger $N$ means more variables. The recent observation of SSCO in a double QD system[10] (three barriers with $N=2$ ) coincides with our view that the relevant variables for an SSCO is two. The two relevant variables construct the embeding surface of the corresponding limit cycle. For simplicity, we shall work on a system with two wells and three barriers.

Before preceding our mapping, we introduce first the vdP equation by finding the governing equation of a nonlinear LCR circuit shown in Fig. 1[11]. $U$ is the applied dc bias. Denote the static voltage across the nonlinear 
resistor $R_{N}$ as $\bar{V}$, and assume $R_{N}$ satisfying the following I-V relation $i=-a V+b V^{3}$, where $a$ and $b$ are two parameters, then the deviation of the voltage across the two ends of the nonlinear resistor, $x=V-\bar{V}$, satisfies the following second-order nonlinear ODE

$$
\ddot{x}+\left(\frac{3 b}{C} x^{2}-\frac{a L-R C}{L C}\right) \dot{x}+\frac{1-a R}{L C} x+\frac{b R}{L C} x^{3}=0,
$$

where $L, C$, and $R$ are the inductance, capacitance, and usual linear resistance, respectively. There is an oscillatory (limit cycle) solution when the nonlinear resistor has an NDC of range of $R C / L<a<1 / R$. Thus, the total differential resistance, $R-1 / a$, is negative. It also means that the usual linear resistance should be small, $R<\sqrt{L / C}$. Under the conditions, $(0,0)$ in $V-\dot{V}$ phase plane is an unstable fixed point[11]. The corresponding equation is the so-called vdP equation[12] that can be written in the form

$$
\ddot{y}+\alpha\left(y^{2}-1\right) \dot{y}+y+\beta y^{3}=0,
$$

where $\alpha, \beta$ are parameters. The type of the limit cycle solution depends on $\alpha$. It changes from sinusoidal for a small $\alpha$ to a relaxation oscillation for a large $\alpha$, meaning that it tends to resemble a series of step functions. This equation is structurally stable such that adding higher order terms shall not change qualitatively its oscillatory solution[7, 11] as long as these higher order terms do not lead to bifurcations. Some explicit examples in the bifurcation analysis can be found in Ref. [7] for the vdP equation. It means that self-sustained oscillations exist in all LCR circuits with an NDC component no matter whether its I-V characteristic follows exactly $i=-a V+$ $b V^{3}$.

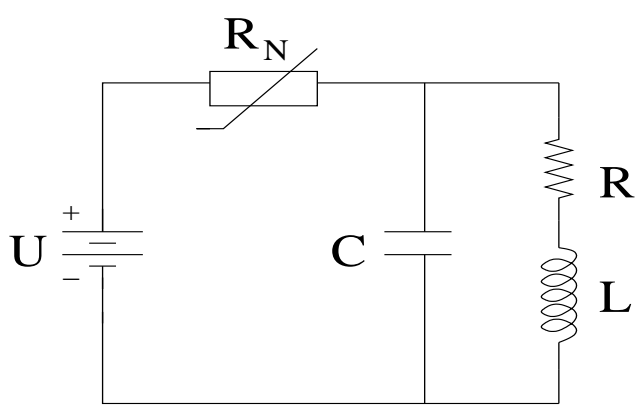

FIG. 1: Schematic drawing of an LCR circuit where $L, C, R$, $R_{N}$ denote the inductance, capacitance, usual linear resistor, and nonlinear resistor of NDC respectively.

We now come back to SSCOs in an SL of two wells and three barriers, the ODEs of $N=2$ become

$$
\begin{aligned}
& J=\dot{V}_{1}+\delta v_{1}\left(V_{1}\right) \\
& J=\dot{V}_{2}+\left(V_{2}-V_{1}+1\right) v_{2}\left(V_{2}\right) \\
& J=-\dot{V}_{1}-\dot{V}_{2}+\left(2-2 V_{2}-V_{1}\right) v_{3}\left(1-V_{2}-V_{1}\right)
\end{aligned}
$$

Assume $\bar{V}_{1}, \bar{V}_{2}$, and $1-\bar{V}_{1}-\bar{V}_{2}$ being the $\mathrm{SSS}$, we can define variables $x_{i}$ as $x_{i} \equiv V_{i}-\bar{V}_{i}$. In order to have an unstable SSS solution[5], two barriers are under NDC. Without losing generality, we can put the first two barriers under NDC and the last one in positive differential resistance regime, and expand their drift velocities in terms of $x_{i}$

$$
\delta v_{i} \equiv v_{i}\left(V_{i}\right)-v_{i}\left(\bar{V}_{i}\right)=-a_{i} x_{i}+b_{i} x_{i}^{3}, \quad i=1,2,3
$$

where $a_{i}$ and $b_{i}$ are expansion coefficients. $a_{1}$ and $a_{2}$ will be positive, giving rise to NDC. Nonlinearity coefficients $b_{i}(i=1,2)$ are necessary for an NDC[11]. We will also take $a_{3}$ to be negative, and $b_{3}=0$.

Since there is no reason why $x_{1}$ or $x_{2}$ should satisfy the vdP equation, (noticing $x_{3}=-x_{1}-x_{2}$ ), we consider the equation obeyed by a general quantity, $Y \equiv x_{1}+g x_{2}$, where $g$ is a parameter to be determined. From Eqs. (6)(7) and after some algebras, one can obtain an equation that $Y$ satisfies

$$
\ddot{Y}+\left(Y^{2}-A\right) \dot{Y}+\omega^{2} Y+f_{h}=0,
$$

where coefficients $A$ and $\omega^{2}$ are

$$
\begin{aligned}
A= & 2\left(\delta a_{1}+\bar{n}_{1} a_{2}+\bar{n}_{2} a_{3}\right) / 3-\left(\bar{v}_{2}+\bar{v}_{3}\right), \\
\omega^{2}= & \frac{1}{3}\left(\delta a_{1} \bar{n}_{1} a_{2}+\delta a_{1} \bar{n}_{2} a_{3}+\bar{n}_{1} a_{2} \bar{n}_{2} a_{3}-\delta a_{1} \bar{v}_{2}\right. \\
& \left.-2 \delta a_{1} \bar{v}_{3}-\bar{n}_{1} a_{2} \bar{v}_{3}-2 \bar{n}_{2} a_{3} \bar{v}_{2}+3 \bar{v}_{2} \bar{v}_{3}\right) . \\
= & \frac{1}{3}\left(\bar{n}_{1} a_{2}-\bar{v}_{2}\right)\left(\delta a_{1}+\bar{n}_{2} a_{3}-\bar{v}_{3}\right) \\
& +\left(\delta a_{1}-\bar{v}_{2}\right)\left(\bar{n}_{2} a_{3}-2 \bar{v}_{3}\right) .
\end{aligned}
$$

$f_{h}$ contains higher order terms in $x_{i}$ 's like $x_{1}^{2}, x_{1} x_{2}, x_{2}^{2}$, etc., and $g$. Without $f_{h}$, Eq. (8) is precisely the vdP equation. A bifurcation occurs whenever an attractor loses its stability. Thus $f_{h}$ in Eq. (8) becomes irrelevant when it does not alter the stability of the limit cycle solution of the vdP equation. For a giving term in the vdP equation, one can use the Liapunov analysis to determine the stability of a limit cycle solution. A practical analysis in the current problem is highly non-trivial since $f_{h}$ in our mapping is described by many parameters including g. In order to see when we can neglect $f_{h}$, it is better to think of $f_{h}$ as a morphology of two-dimensional surface with "mountains", "lakes", and "plains". Obviously, $f_{h}$ can be treated as a constant if it is a "plain" around $\left(x_{1}=0, x_{2}=0\right)$. Then, Eq. (8) is just a vdP equation. In general, in the case that terms in $f_{h}$ do not cause any bifurcation, $f_{h}$ can be dropped as long as we are interested in the self-sustained oscillation (limit cycle) solutions only, and Eq. (8) is the same as the vdP equation. The possible occurrence of this case is guaranteed by the structural stability of the vdP equation[7]. However, $f_{h}$ should be important, and may cause bifurcations[11] if $\left(x_{1}=0, x_{2}=0\right)$ is among a rough "mountain". Many other interesting solutions are possible[11]. When $f_{h}$ will be important is not the concern of current work although 
it will be important for other type of solutions like strange attractors.

Comparing Eq. (8) with Eq. (4), the vdP equation of an LCR circuit, the product of the effective capacitance and the effective inductance is inversely proportional to $\omega^{2}$, i.e.,

$$
L C \propto \frac{1}{\omega^{2}}
$$

The Eq. (8) has an oscillatory solution only for $A>0$, $\omega^{2}>0$. Thus the total differential resistance is negative, $\delta a_{1}+\bar{n}_{1} a_{2}+\bar{n}_{2} a_{3}>\left(\bar{v}_{2}+\bar{v}_{3}\right) \geq 0$. In certain sense, the effective inductance is the consequence of the NDC.

The mapping was achieved by assuming that there are two potential barriers under NDC regime. This is consistent with general stability conditions[5], and our early result that the SSCOs originate from the generation of a limit cycle around an unstable SSS[6]. The mapping is expected to hold for large $N$ in the SSCO regime. This can be understood in terms of phase flow diagram. The dynamics will quickly bring a system into the $2 \mathrm{D}$ phase surface where the limit cycle is embedded in. Thus, there are only two quantities are relevant, and rest of them are irrelevant variables, as long as one concerns an SSCO. In this mapping, however, we have not provided the conditions under which such an unstable SSS exists. Ex- perimentally, SSCO appears only at right doping, temperature, and other system parameters. It is easy to be observed in an SL of around 30 wells. Thus the conditions must depend on the number of wells and other system parameters. It would be interesting to know how the size of SSCOs regime depends on number of wells.

We would like to point out that the dynamics of SLs is equivalent to a vdP equation as long as one is only interested in the SSCO. However, one should understand that our mapping does not deny other more complicated timedependent electric current such as quasi-periodic behaviors and chaos[13].

In summary, we have mapped a discrete drift model widely used for SSCOs in SLs into the vdP equation. We provide a theoretical base of why a self-sustained oscillation can in principle be modelled by a second-order ODE: A limit cycle is embedded topologically in a two dimensional surface. In terms of flow diagram in the phase space, there are only two relevant variables that span the surface for the limit cycle. Its dynamics is then determined by a second ODE. We obtain an equivalent circuit for SLs in the SSCO regime, in which the effective inductance is originated from the negative differential conductance.

This work is supported by UGC, Hong Kong, through grants No. 603403. Y.P. Wang is supported by the NSF of China. Y.Q. Wang is supported by CAS.
[1] L. Esaki, and R. Tsu, IBM J. Res. Dev. 14, 61 (1970); R. Tsu, and L. Esaki, Appl. Phys. Lett. 22, 562 (1973).

[2] L. L. Chang, L. Esaki, and R. Tsu, Appl. Phys. Lett. 24, 593 (1974); L. Esaki and L.L. Chang, Phys. Rev. Lett. 33, 495 (1974).

[3] S. H. Kwok, T. B. Norris, L. L. Bonilla, J. Galán, J. A. Cuesta, F. C. Martínez, J. M. Molera, H. T. Grahn, K. Ploog, R. Merlin, Phys. Rev. B 51, 10171 (1995); H. T. Grahn, J. Kastrup, K. Ploog, L. L. Bonilla, J. Galán, M. Kindelan, and M. Moscoso, Jpn. J. Appl. Phys. 34, 4526 (1995); J. Kastrup, R. Klann, H. T. Grahn, K. Ploog, L. L. Bonilla, J. Galán, M. Kindelan, M. Moscoso, R. Merlin, Phys. Rev. B 52, 13761 (1995).

[4] J. N. Wang, B. Q. Sun, X. R. Wang, Y. Q. Wang, W. K. Ge, and H. L. Wang, Appl. Phys. Lett. 75, 2620 (1999); X. R. Wang, J. N. Wang, B. Q. Sun, and D. S. Jiang, Phys. Rev. B 61, 7261 (2000).

[5] X. R. Wang, and Q. Niu, Phys. Rev. B. 59, R12755 (1999); X. R. Wang, et al., cond-mat/0211306.

[6] Z. Z. Sun, H. T. He, J. N. Wang, Shi-dong Wang, and X. R. Wang, Phys. Rev. B (to appear); cond-mat/0304648.

[7] Lecture Notes on Nonlinear Vibrations, R. H. Rand, Ver- sion 40, http://www.tam.cornell.edu/randdocs, Cornell University.

[8] N. Zou, M. Willander and K.A. Chao, Phys. Rev. B. 50, 4980 (1994).

[9] L. L. Bonilla, J. Galán, J. A. Cuesta, F. C. Martínez, and J. M. Molera, Phys. Rev. B 50, 8644 (1994); F. Prengel, A. Wacker, and E. Schöll, Phys. Rev. B 50, 1705 (1994); R. Aguado, G. Platero, M. Moscoso, and L. L. Bonilla, Phys. Rev. B 55, R16053 (1997); J. Kastrup, R. Hey, K. H. Ploog, H. T. Grahn, L. L. Bonilla, M. Kindelan, M. Moscoso, A. Wacker, J. Galán, Phys. Rev. B 55, 2476 (1997).

[10] K. Ono and S. Tarucha , Phys. Rev. Lett. 92, 256803 (2004).

[11] "Practical Bifurcation and Stability Analysis", R. Seydel, Springer-Verlag, New York, 1994.

[12] The standard van der Pol equation is defined as Eq. (6) with $\beta=0$.

[13] Y. Zhang et al., Phys. Rev. Lett. 77, 3001 (1996); K. J. Luo, H. T. Grahn, K. H. Ploog, and L. L. Bonilla, Phys. Rev. Lett. 81, 1290 (1998). 